University of Wollongong

Research Online

Australian Institute for Innovative Materials -

Papers

Australian Institute for Innovative Materials

$1-1-2013$

Spin switching and magnetization reversal in single-crystal $\mathrm{NdFeO3}$

S J. Yuan

Shanghai University

W Ren

Shanghai University

F Hong

University of Wollongong, fh640@uowmail.edu.au

Y B. Wang

Shanghai University

J C. Zhang

Shanghai University, jz744@uow.edu.au

See next page for additional authors

Follow this and additional works at: https://ro.uow.edu.au/aiimpapers

Part of the Engineering Commons, and the Physical Sciences and Mathematics Commons

Research Online is the open access institutional repository for the University of Wollongong. For further information contact the UOW Library: research-pubs@uow.edu.au 


\title{
Spin switching and magnetization reversal in single-crystal $\mathrm{NdFeO3}$
}

\begin{abstract}
We report an experimental and computational study of single-crystal $\mathrm{NdFeO3}$, which features two inequivalent magnetic sublattices, namely, Fe and $\mathrm{Nd}$ sublattices that are coupled in an antiparallel fashion. This paper reveals that a strong interaction between $3 \mathrm{~d}$ and $4 \mathrm{f}$ electrons of the two sublattices along with a spin-lattice coupling drives an extremely interesting magnetic state that is highly sensitive to the orientation and history of weak magnetic field. The following phenomena are particularly remarkable: (1) sharply contrasting magnetization $\mathrm{M}(\mathrm{T})$ along the a and c axes; (2) a first-order spin switching along the a axis below $29 \mathrm{~K}$ when the system is zero-field-cooled; and (3) a progressive magnetization reversal when the system is field-cooled. The intriguing magnetic behavior is captured in our first-principles density functional theory calculations.
\end{abstract}

\section{Keywords}

crystal, single, reversal, 3 , magnetization, ndfeo, switching, spin

Disciplines

Engineering | Physical Sciences and Mathematics

\section{Publication Details}

Yuan, S., Ren, W., Hong, F., Wang, Y. B., Zhang, J. C., Bellaiche, L., Cao, S. X. \& Cao, G. (2013). Spin switching and magnetization reversal in single-crystal NdFeO 3. Physical Review B - Condensed Matter and Materials Physics, 87 (18), 184405-184405-6.

\section{Authors}

S J. Yuan, W Ren, F Hong, Y B. Wang, J C. Zhang, L Bellaiche, S X. Cao, and G Cao 


\title{
Spin switching and magnetization reversal in single-crystal $\mathrm{NdFeO}_{3}$
}

\author{
S. J. Yuan, $,{ }^{1,2,}, \dagger$ W. Ren, ${ }^{1,3}$ F. Hong, ${ }^{4}$ Y. B. Wang, ${ }^{1}$ J. C. Zhang, ${ }^{1}$ L. Bellaiche, ${ }^{3}$ S. X. Cao, ${ }^{1}$ and G. Cao ${ }^{2}$ \\ ${ }^{1}$ Department of Physics, Shanghai University, Shanghai 200444, China \\ ${ }^{2}$ Department of Physics and Astronomy and Center for Advanced Materials, University of Kentucky, Lexington, Kentucky 40506, USA \\ ${ }^{3}$ Physics Department and Institute for Nanoscience and Engineering, University of Arkansas, Fayetteville, Arkansas 72701, USA \\ ${ }^{4}$ Institute for Superconducting and Electronic Materials, University of Wollongong, New South Wales 2519, Australia
}

(Received 14 December 2012; revised manuscript received 25 April 2013; published 8 May 2013)

\begin{abstract}
We report an experimental and computational study of single-crystal $\mathrm{NdFeO}_{3}$, which features two inequivalent magnetic sublattices, namely, $\mathrm{Fe}$ and $\mathrm{Nd}$ sublattices that are coupled in an antiparallel fashion. This paper reveals that a strong interaction between $3 d$ and $4 f$ electrons of the two sublattices along with a spin-lattice coupling drives an extremely interesting magnetic state that is highly sensitive to the orientation and history of weak magnetic field. The following phenomena are particularly remarkable: (1) sharply contrasting magnetization $M(T)$ along the $a$ and $c$ axes; (2) a first-order spin switching along the $a$ axis below $29 \mathrm{~K}$ when the system is zero-field-cooled; and (3) a progressive magnetization reversal when the system is field-cooled. The intriguing magnetic behavior is captured in our first-principles density functional theory calculations.
\end{abstract}

DOI: 10.1103/PhysRevB.87.184405

PACS number(s): 75.50.Ee, 71.15.Mb, 75.30.Gw

\section{INTRODUCTION}

Emerging technologies are increasingly predicated upon the discovery of novel functional materials that exhibit striking physical properties such as spin switching and magnetization reversal. Materials such as rare-earth-based orthoferrites $R \mathrm{FeO}_{3}$ ( $R=$ rare-earth element) are clearly of fundamental interest and technological importance for potential applications such as ultrafast photomagnetic recording, laser-induced ultrafast spin reorientation, ${ }^{1}$ excitation of precession, ${ }^{2}$ nonthermal spin dynamics, ${ }^{3}$ inertia-driven spin switching, ${ }^{4}$ and ambient multiferroics. ${ }^{5}$ In particular, the spin dynamics of these spin-canted antiferromagnets dictated by the exchange interaction is approximately two orders of magnitude faster than those in metallic or insulating ferromagnets. ${ }^{2}$ Certainly, looming in the future are possible device applications in such a class of materials. ${ }^{6-10}$ However, before this is vigorously pursued, a better knowledge of their respective properties needs to be established.

$R \mathrm{FeO}_{3}$ adopts an orthorhombic distortion with space group Pbnm and features two inequivalent magnetic sublattices, namely, $4 f$ electron based $R$ sublattice and $3 d$ electron based Fe sublattice that are coupled in an antiparallel fashion. Noncollinear antiferromagnetism in the Fe sublattice gives rise to weak ferromagnetism ${ }^{11,12}$ in the orthoferrites ${ }^{13,14}$ at relatively high temperatures, while the $R$ sublattice typically orders antiferromagnetically at much lower temperatures [note that there are a few noticeable exceptions for which the $R$ sublattice magnetically orders at relatively high temperatures, e.g. $\sim 100 \mathrm{~K}$ or higher in $\mathrm{SmFeO}_{3}$ (Refs. 5 and 15)]. Since the exchange interaction of $3 d$ electrons is considerably stronger than that of $4 f$ electrons, the two sublattices exhibit different temperature dependence; and novel phenomena often emerge from the strong competition between the two magnetic sublattices. Nevertheless, the resultant magnetization $M$ dictated by the strong coupling between $3 d$ and $4 f$ electrons includes contributions from both $R$ and Fe sublattices: ${ }^{14,16}$

$$
M=M_{0}+\chi_{R} H_{0},
$$

where $M_{0}$ is the magnetization of the Fe sublattice, $\chi_{R}$ is the paramagnetic susceptibility of the $R$ sublattice, and $H_{0}$ is the exchange field at the $R$ sublattice generated by the magnetic moment of the Fe sublattice. ${ }^{14,16}$ When $M_{0}$ and $\chi_{R}$ have a different sign and follow different temperature dependence, Eq. (1) adequately explains the following phenomena observed in $R \mathrm{FeO}_{3}$ : (i) the compensation temperature $T_{\text {comp }}$ at which $M=0$ when $\left|\chi_{R} H_{0}\right|=\left|M_{0}\right|$; and (ii) the magnetization reversal when one of the two sublattices dominates the other at temperatures above $T_{\text {comp }}$. As a matter of fact, this magnetization reversal was indeed observed in $R \mathrm{FeO}_{3}$ where $R=\mathrm{Er}, \mathrm{Tm}, \mathrm{Sm},{ }^{14-16}$ and other antiferromagnets, such as orthochromites, ${ }^{17,18}$ orthovanadates, ${ }^{19,20}$ and manganites. ${ }^{21-23}$

The magnetization reversal, in which the direction of magnetization is opposite to that of the applied magnetic field, is not energetically favorable. The occurrence of the magnetization reversal in $R \mathrm{FeO}_{3}$ is a clear manifestation that the magnetic ground state must be unstable and susceptible to small perturbations due chiefly to the strong competition between $3 d$ and $4 f$ electrons. Given the circumstance described above, it almost guarantees that extraordinary phenomena will occur when the magnetic state is probed in external magnetic field applied along different principal crystalline axes. It is this realization that has motivated us to investigate the magnetic properties of $\mathrm{NdFeO}_{3}$, a prototype of $R \mathrm{FeO}_{3}$, both experimentally and computationally. This paper reveals a strong interaction between the Fe and Nd sublattices; this interaction along with spin-lattice coupling drives an extremely unstable magnetic ground state that gives rise to intriguing phenomena such as unusually large magnetic anisotropy, magnetization reversal, and spin switching when the magnetic state is probed at low magnetic fields. It is particularly intriguing that when $\mathrm{NdFeO}_{3}$ is zero-field-cooled, the $a$ axis magnetization $M_{a}(T)$ undergoes a first-order transition leading to an abrupt, complete magnetization reversal, i.e. $M_{a}(T)$ changes from -0.3 to $0.3 \mu_{B} /$ f.u. at a characteristic temperature $T_{\text {jump }}=$ 29 K. Furthermore, the isothermal magnetization for $M_{a}(H)$ also features a first-order spin switching at a critical field $H_{c}$ that decreases with increasing temperature. These novel phenomena that are unique to $\mathrm{NdFeO}_{3}$ constitute the central findings of this paper. The first-order transition in both $M_{a}(T)$ and $M_{a}(H)$ occurring at such a low temperature and magnetic 
field manifests an exceptionally delicate magnetic ground arising from the strong competition between the $\mathrm{Nd}$ and $\mathrm{Fe}$ sublattices. It is this magnetic ground state that we seek to understand.

\section{EXPERIMENTAL AND COMPUTATIONAL DETAILS}

Single-crystal $\mathrm{NdFeO}_{3}$ was grown using the floating zone technique. Details of single-crystal synthesis and characterization are described elsewhere. ${ }^{24,25}$ The compositional homogeneity and crystal morphology were analyzed by $\mathrm{x}$-ray diffraction (XRD), scanning electron microscopy (SEM), and energy-dispersive X-ray spectroscopy (EDX). All results confirmed the high homogeneity of all crystals studied. Measurements of magnetization as a function of temperature and magnetic field were performed using the Quantum Design Physical Property Measurement System, PPMS-9. Zero-fieldcooling (ZFC) and field-cooling (FC) processes were used to acquire the temperature dependence of the magnetization. The measurements were performed during the warming process. The cooling and measuring fields are both 100 Oe, unless explicitly specified.

We also carried out first-principles density functional theory (DFT) calculations on the basis of the generalized gradient approximation (GGA) method. ${ }^{26}$ The projector augmented wave (PAW) pseudopotential ${ }^{27}$ was implemented in the Vienna $A b$ initio Simulation Package ${ }^{28}$ by explicitly treating 14 valence electrons for $\mathrm{Fe}\left(3 p^{6} 3 d^{7} 4 s^{1}\right)$, and 6 for oxygen $\left(2 s^{2} 2 p^{4}\right)$. Two different PAW pseudopotentials for $\mathrm{Nd}$ were used: one for which $\mathrm{Nd}$ has 11 valence electrons $\left(5 s^{2} 5 p^{6} 5 d^{1} 6 s^{2}\right)$, and a second one for which 14 valence electrons were simulated for $\mathrm{Nd}$ ions $\left(4 f^{3} 5 s^{2} 5 p^{6} 5 d^{1} 6 s^{2}\right)$ that were allowed to carry the magnetic moments. Comparing results between these two cases can thus help us in better understanding the precise effect of the $4 f$ electrons of $\mathrm{Nd}$ ions on physical properties of $\mathrm{NdFeO}_{3}$. We have tested GGA, Perdew-Burke-Ernzerhof revised for solids (PBEsol), and Perdew-Burke-Ernzerhof (PBE) functionals ${ }^{29,30}$ to relax the structure, and found that the calculated lattice parameters are reasonably close to experimental ones. When performing the structural relaxation, we calculated the forces on the ions, to be converged to $0.005 \mathrm{meV} / \AA$. The structural relaxation does not significantly change the calculated magnetic moments, but the PBE/PBEsol functionals (and Hubbard $U^{31}$ ) can underestimate the magnetic moments with respect to experiment. This is also why we choose the GGA functional, which gives values closer to the measured magnetic moments. Moreover, from our experience, it seems that the electronic convergence is easier to be achieved when using GGA. We have also tested calculations both with and without Hubbard $U$ applied to the $\mathrm{Nd} 4 f$ and $\mathrm{Fe}$ $3 d$. We found that when we turned on $U(U=6 \mathrm{eV}$ for $\mathrm{Nd}$ and $U=4 \mathrm{eV}$ for $\mathrm{Fe}$ ) the calculated magnetic moments were suppressed in magnitude by a factor of three. When turning off $U$, we still obtained a nonmetallic electronic structure with Fermi level located inside the band gap. We chose to mimic the orthorhombic Pbnm space group, by selecting a 20 -atom cell and by adopting the lattice parameters experimentally found at both high and low temperatures (290 and $1.5 \mathrm{~K}$, respectively). ${ }^{32}$ All DFT calculations were performed using (i) a $6 \times 6 \times 4$ Monkhorst-Pack k-point mesh centered at $\Gamma,{ }^{33}$ (ii) a 500-eV plane-wave cutoff energy, and (iii) noncollinear magnetism and spin-orbit coupling were included in the simulations, and symmetry was switched off during the computations.

\section{RESULTS AND DISCUSSION}

One of major characteristics of $\mathrm{NdFeO}_{3}$ is the strong magnetic anisotropy that leads to starkly different temperature dependence of $M_{a}(T)$ and $M_{c}(T)$, as shown in Fig. 1. The anomaly at $T_{S R}=170 \mathrm{~K}$ marks an onset of a spin reorientation of the $\mathrm{Fe}$ sublattice that completes at $107 \mathrm{~K}$ (see shaded region in Fig. 1). ${ }^{10}$ This spin reorientation leads to fascinating behavior at low temperatures. It is apparent that $M_{a}(T)$ exhibits intriguing behavior that is highly sensitive to the history of the magnetic field at low temperatures $(T<29 \mathrm{~K})$ whereas $M_{c}(T)$ remains essentially unchanged in this temperature region.

We therefore focus our attention on $M_{a}(T)$ at low temperatures. Here, $M_{a}(T)$ exhibits drastically different temperature dependence at low temperatures when measured using ZFC and FC processes, as shown in Figs. 1(a) and 1(b). Complex behavior resulting from competing $\mathrm{Fe}$ and $\mathrm{Nd}$ sublattices at low temperatures deserves a close examination.
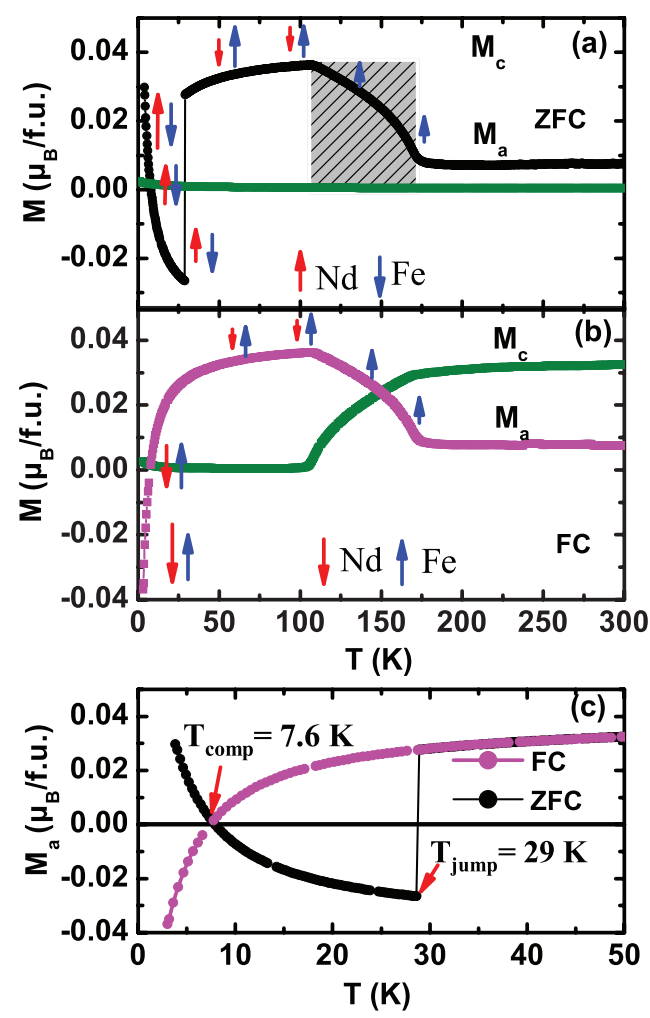

FIG. 1. (Color online) Temperature dependence of the magnetization $M(T)$ of $\mathrm{NdFeO}_{3}$ single crystal along $a$ and $c$ axes measured in (a) ZFC and (b) FC processes; (c) the magnified view of the ZFC and FC $M(T)$ curves at low temperatures. The cooling and measuring fields are $100 \mathrm{Oe}$. The arrows in (a) and (b) represent the evolution of the effective magnetic moment of $\mathrm{Fe}$ (in blue) and $\mathrm{Nd}$ (in red) ions. The shadow in (a) marks the spin reorientation region of the Fe sublattice; the inset in (a) shows the image of the as-grown single crystal $\mathrm{NdFeO}_{3}$. 

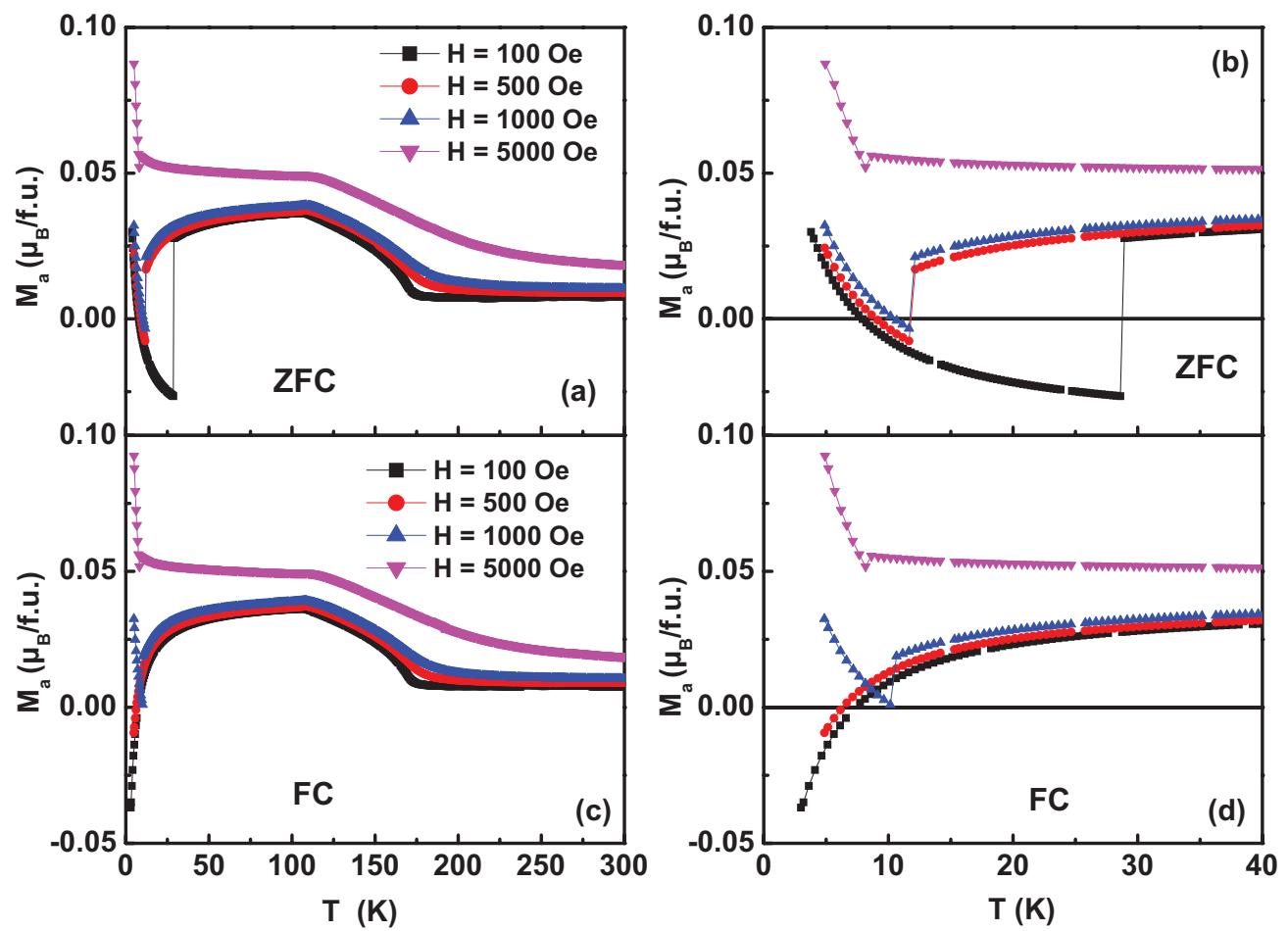

FIG. 2. (Color online) Temperature dependence of the magnetization $M(T)$ along the $a$ axis for (a) and (b) the ZFC sequence and (c) and (d) the FC sequence under various applied magnetic fields. (b) and (d) show the magnified view of the curves near the compensation point in (a) and (c), respectively.

In the ZFC case, with increasing temperature, $M_{a}(T)$ decreases and then reaches zero at the compensation temperature $T_{\text {comp }}=7.6 \mathrm{~K}$ before it becomes negative, as shown in Figs. 1(a) and 1(c). The decrease in $M_{a}(T)$ below $T_{\text {comp }}$ is attributed to the fact that the effective moment of the $\mathrm{Nd}$ sublattice decreases faster than that of the antiparallel $\mathrm{Fe}$ sublattice when temperature rises [see schematic arrows in Fig. 1(a)]; the moment of the two sublattices becomes equal in magnitude but opposite in direction at $T_{\text {comp }}(=7.6 \mathrm{~K})$, which explains the vanishing $M_{a}$ at that temperature. For $T_{\text {comp }}<$ $T<29 \mathrm{~K}$, the moment of the Fe sublattice dominates that of the Nd sublattice, resulting in the negative $M_{a}$, as shown in Fig. 1(a).

Remarkably, $M_{a}(T)$ exhibits a first-order transition at $T_{\text {jump }}=29 \mathrm{~K}$ that leads to a sign change of the magnetization, i.e. $M_{a}(T)$ jumps from -0.027 to $+0.028 \mu_{B}$. Such a firstorder transition is likely due to a spontaneous spin reversal of the Fe and Nd sublattices, as schematically shown in Fig. 1(a). The net ferromagnetic vectors of the $\mathrm{Nd}$ and $\mathrm{Fe}$ sublattice are antiparallel to each other, with the ferromagnetic vector of the Nd sublattice being likely aligned with the applied magnetic field $H$, and both follow vastly different temperature dependence. Thus, increasing temperature facilitates a magnetization reversal below $T<T_{\text {jump }}(=29 \mathrm{~K})$ since the magnetization of the $\mathrm{Nd}$ sublattice weakens much faster than that of the Fe sublattice. When temperature approaches $\mathrm{T}_{\text {jump }}$, both of the sublattices undergo a spin-flip transition to a more energetically favorable state with the direction of $M_{a}(T)$ aligned with that of $H$; and $M_{a}(T)$ is now dominated by the Fe sublattice. This explains the first-order transition in $M_{a}(T)$ [Figs. 1(a) and 1(c)]. Indeed, $T_{\text {jump }}$ decreases as $H$ increases, leading to a decreasing absolute value of the negative $M_{a}(T)$ from $-0.027 \mu_{B}$ at $H=100$ Oe to $-0.003 \mu_{B}$ at $H=$ 1000 Oe. When $H$ is 5000 Oe or stronger, $T_{\text {jump }}$ vanishes, and $M_{a}(T)$ is aligned with the direction of $H$, as shown in Figs. 2(a) and 2(b). The fact that weak magnetic fields can induce such drastic changes in $M_{a}(T)$ clearly highlights an extremely delicate magnetic ground state at low temperatures.

When $M_{a}(T)$ is measured in a FC sequence, spins of both sublattices are "locked up", thus a stronger field is required for the first-order spin-flip transition to occur; indeed, the transition at $T_{\text {jump }}$ becomes much weaker and essentially vanishes at $H=5000$ Oe, as shown in Figs. 2(c) and 2(d). The fact that all these differences between the ZFC and FC $M_{a}(T)$ occur below 5000 Oe corroborates a highly unstable magnetic state due to the strong competition of the two magnetic sublattices.

The isothermal magnetization $M_{a}(H)$ is also characterized by a first-order spin switching at weak magnetic fields, as shown in Fig. 3. The unsaturated $M_{a}(H)$ suggests an underlying antiferromagnetic (AFM) state; ${ }^{34}$ however, the first-order spin switching is apparently due to spin canting of the Fe sublattice. When $T$ approaches $T_{\text {comp }}(=7.6 \mathrm{~K})$, no hysteresis loop occurs because $M_{a}$ is essentially zero, as discussed above (Fig. 1). The magnitude of the remnant magnetization at $5 \mathrm{~K}\left(0.019 \mu_{B}\right)$ is smaller than that at $20 \mathrm{~K}\left(0.027 \mu_{B}\right)$. Such temperature-induced reduction of the remnant magnetization shown in Fig. 1(a) sharply contrasts that in conventional magnets possessing a spin-canted structure such as $\mathrm{BiFeO}_{3} .{ }^{11}$ It originates from the strongly temperature-dependent ferromagnetic vectors of the $\mathrm{Nd}$ and $\mathrm{Fe}$ sublattices that are antiparallel; note that 


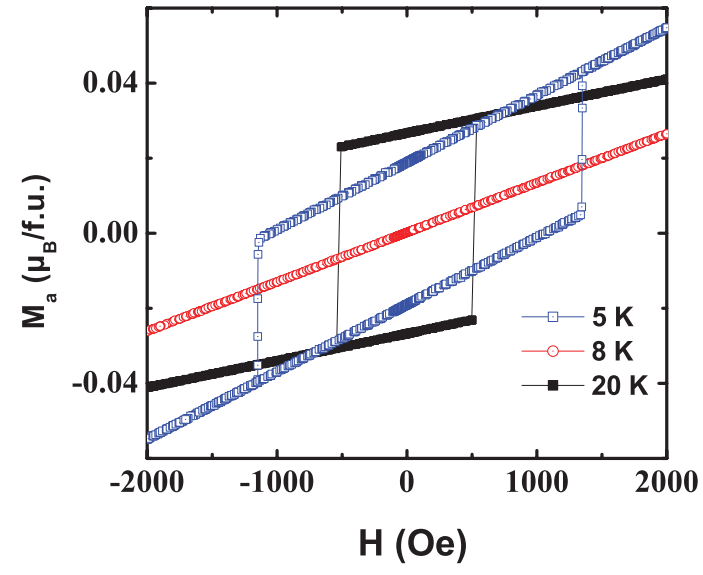

FIG. 3. (Color online) The isothermal magnetization along the $a$ axis as a function of magnetic field. The three investigated temperatures are 5,8 , and $20 \mathrm{~K}$.

5 and $20 \mathrm{~K}$ are situated below and above $T_{\text {comp }}=7.6 \mathrm{~K}$, respectively.

We also examine the magnetic state by performing firstprinciples calculations. At $T>T_{S R}(=170 \mathrm{~K})$, the Fe sublattice adopts a $G$-type AFM vector with the resulting AFM vector aligned along the $a$ axis, which is denoted as the $\Gamma_{4}$ spin state or $\left(G_{x}, F_{z}\right){ }^{35}$ It is established that the spin-lattice interaction dictates the spin configuration and the tilting of Fe-O octahedra [see illustration in Fig. 4(a) made by Vesta software $^{36}$ in the Pbnm structural phase along with this AFM vector facilitates a Dzyaloshinsky-Moriya (DM) spin canting or weak ferromagnetic (FM) vector that is aligned along the $c$ axis, ${ }^{12}$ as shown in Fig. 1. Here, the $x$ and $z$ axes are chosen along $a$ and $c$ axes, respectively. Our $0 \mathrm{~K}$ first-principles calculations for the $\Gamma_{4}$ spin state, without considering magnetism due to the Nd sublattice, yields the following magnetic moments in Bohr magneton $\mu_{B}$ for the four Fe ions, as shown in Fig. 4: $S_{\mathrm{Fe} 1}=(3.714,0.028,0.025), S_{\mathrm{Fe} 2}=$ $(-3.714,-0.028,0.023), S_{\mathrm{Fe} 3}=(-3.714,0.027,0.023)$, and $S_{\mathrm{Fe} 4}=(3.714,-0.028,0.025)$. The computations predict the existence of a net magnetization of $0.023 \mu_{B}$ for the $\Gamma_{4}$ spin state, which is reasonably consistent with the experimental result of $M_{c} \sim 0.03 \mu_{B}$ displayed in Fig. 1(a) (especially when it is interpolated to $0 \mathrm{~K}$ ). In essence, the computational results endorse the $\Gamma_{4}$ spin state for $T>T_{S R}$, where the weak ferromagnetism arises from the Fe sublattice.

The ferromagnetic moment clearly undergoes a gradual spin reorientation from the $c$ to the $a$ axis as temperature decreases from 170 to $107 \mathrm{~K}$ in the Fe sublattice, as illustrated in Fig. 1; this spin reorientation marks a crossover from the $\Gamma_{4}$ to the $\Gamma_{2}$ spin state. The $\Gamma_{2}$ spin state or $\left(G_{z}, F_{x}\right)$ indicates that the Fe sublattice still retains the $G$-type antiferromagnetism but with the underlying antiferromagnetic vector rotated to the $c$ axis from the previous $a$ axis. Our $0 \mathrm{~K}$ first-principles calculations for the $\Gamma_{2}$ spin state (neglecting again magnetism due to the $\mathrm{Nd}$ sublattice) produces the following magnetic moments in $\mu_{B}: S_{\mathrm{Fe} 1}=(0.026,0.024,-3.710), S_{\mathrm{Fe} 2}=(0.025$, $0.024,3.710), S_{\mathrm{Fe} 3}=(0.025,-0.024,+3.710), S_{\mathrm{Fe} 4}=(0.026$, $-0.024,-3.710)$. The net magnetization for the Fe sublattice amounts to $0.026 \mu_{B}$, which is also reasonably close to the experimental value (and its interpolation to $0 \mathrm{~K}$ ) of $M_{a} \sim$ $0.035 \mu_{B}$ (Fig. 1) in the vicinity of $107 \mathrm{~K}$ where the crossover is completed.

As temperature further decreases, the exchange interaction $H_{o}$ becomes increasingly significant and causes a spin alignment of the weak ferromagnetic vector in the $\mathrm{Nd}$ sublattice that is antiparallel to that of the Fe sublattice; and both the sublattices obey starkly different temperature dependence with the $\mathrm{Nd}$ sublattice having stronger temperature dependence.
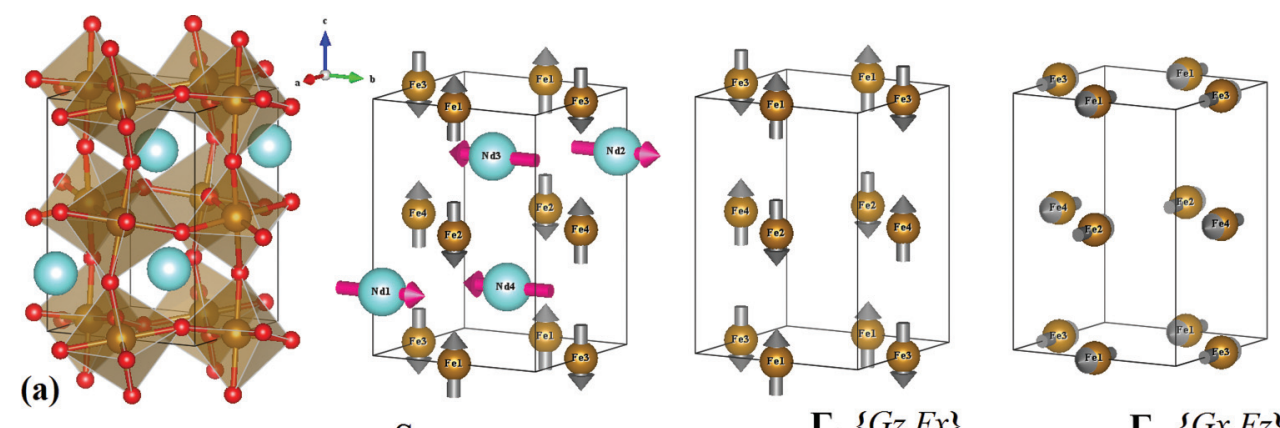

(b)
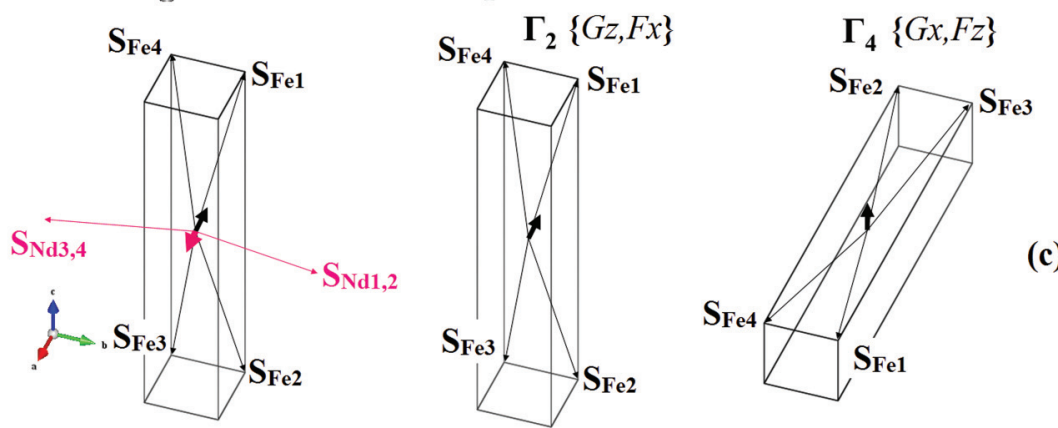

FIG. 4. (Color online) Crystal structure, magnetic configurations of orthorhombic $\mathrm{NdFeO}_{3}$. (a) A unit-cell crystal structure of $\mathrm{NdFeO}_{3}$ in Pbnm setting (Cyan: Nd, Brown: Fe, Red: O). (b) and (c) Schematic spin structures for low temperature, $\Gamma_{2}$ and $\Gamma_{4}$ configurations. Note the arrows in (c) are exaggerated for showing clearly the weak ferromagnetisms due to the canted antiferromagnetic orders. 
In the above calculations, we start with $\left(G_{z}, F_{x}\right)$ or the $\Gamma_{2}$ spin state of the Fe sublattice but use pseudopotentials for which the Nd sublattice produces no magnetic order. On the other hand, we now allow the $\mathrm{Nd}$ sublattice to adopt magnetism using a different set of pseudopotentials via the incorporation of $4 f$ electrons in the valence shells. After convergence is reached, the $\mathrm{Nd}$ sublattice possesses a $C$-type AFM order with the AFM vector aligned with the $b$ axis and a FM component collinear with the $a$ axis. The final spin configuration features a coexistence ${ }^{37}$ of both the $\left(C_{y}, F_{x}\right)$ spin structure for the $\mathrm{Nd}$ sublattice and the $\left(G_{z}, F_{x}\right)$ spin configuration for the Fe sublattice. It is interesting to know that starting the calculations with the $\left(C_{y}, F_{x}\right)$ spin structure for the Nd sublattice and no magnetic ordering for the Fe sublattice does not provide the $\left(G_{z}, F_{x}\right)$ orderings for the $\mathrm{Fe}$ sublattice after convergence is reached. Such results therefore strongly suggest that the occurrence and type of magnetic orderings of the $4 f$ electrons of the Nd sublattice are induced by the $3 d$ electrons of the Fe sublattice, while the ordering existing in the Fe sublattice has nothing to do with $4 f$ electrons and their interactions with $3 d$ electrons. The results of these calculations therefore confirm a strong exchange interaction $H_{o}$ between the two sublattices [see Eq. (1)], and it is this $H_{o}$ that induces the magnetic ordering in the $\mathrm{Nd}$ sublattice at low temperatures.

Moreover, the calculations of the phase possessing the $\left(C_{y}, F_{x}\right)$ arrangement for the $\mathrm{Nd}$ sublattice and the $\left(G_{z}, F_{x}\right)$ configurations for the Fe sublattice yield a weak ferromagnetic vector associated with the $\mathrm{Nd}$ sublattice that has a positive component of $+0.062 \mu_{B}$ along the $a$ axis, while the ferromagnetic vector in the Fe sublattice has a negative component of $-0.015 \mu_{B}$ along that $a$ axis (see Fig. 4). Therefore, the first-principles calculations indicate that the total positive magnetization of $\sim 0.03 \mu_{B}$ displayed in Fig. 1(a) (for the lowest temperatures) originates from the facts that the $\mathrm{Nd}$ and Fe sublattices have opposite ferromagnetic vectors and that the ferromagnetic vector of the $\mathrm{Nd}$ sublattice is larger in magnitude than that of the Fe sublattice at low temperatures.

\section{CONCLUSIONS}

We have studied the magnetic properties of single-crystal $\mathrm{NdFeO}_{3}$ both experimentally and computationally. All results indicate that the strong interaction between the two sublattices or $d$ and $f$ electrons along with the spin-lattice coupling renders an extremely unstable magnetic state; it is this instability that is highly susceptible to small perturbations and generates the intriguing phenomena such as the magnetization reversal and first-order spin switching, which constitute the central findings of this study.

\section{ACKNOWLEDGMENTS}

This work is supported by the National Natural Science Foundation of China (NSFC, Nos. 50932003, 11274221, 11274222, 11074163), the Eastern Scholar Professorship at Shanghai Institutions of Higher Education, Shanghai Municipal Education Commission, Shanghai Shuguang Program (12SG34), and the Research Innovation Fund of the Shanghai Education Committee (No.12YZ018). L.B. and W.R. thank the financial support of ARO Grant W911NF-12-1-0085. They also acknowledge the Department of Energy, Office of Basic Energy Sciences, under Contract ER-46612, ONR grants N00014-11-1-0384, N00014-12-1-1034, and N00014-08-10915, and NSF grants DMR-1066158 and DMR-0701558 for discussions with scientists supported by these grants. Some computations were also made possible thanks to the MRI Grant 0722625 from NSF, Shanghai Supercomputer Center, the ONR Grant N00014-07-1-0825 (DURIP) and a Challenge Grant from the Department of Defense. S.J.Y. and G.C. acknowledge NSF support through grants DMR-0856234 and EPS-0814194.

\footnotetext{
*Visiting professor at the University of Kentucky

${ }^{\dagger}$ Corresponding author: shujuanyuan@shu.edu.cn

${ }^{1}$ J. A. de Jong, A. V. Kimel, R. V. Pisarev, A. Kirilyuk, and T. Rasing, Phys. Rev. B 84, 104421 (2011).

${ }^{2}$ A. V. Kimel, A. Kirilyuk, A. Tsvetkov, R. V. Pisarev, and T. Rasing, Nature 429, 850 (2004).

${ }^{3}$ A. V. Kimel, A. Kirilyuk, P. A. Usachev, R. V. Pisarev, A. M. Balbashov, and T. Rasing, Nature 435, 655 (2005).

${ }^{4}$ A. V. Kimel, B. A. Ivanov, R. V. Pisarev, P. A. Usachev, A. Kirilyuk, and T. Rasing, Nat. Phys. 5, 727 (2009).

${ }^{5}$ J.-H. Lee, Y. K. Jeong, J. H. Park, M.-A. Oak, H. M. Jang, J. Y. Son, and J. F. Scott, Phys. Rev. Lett. 107, 117201 (2011).

${ }^{6}$ Y. Tokunaga, N. Furukawa, H. Sakai, Y. Taguchi, T.-h. Arima, and Y. Tokura, Nat. Mater. 8, 558 (2009).

${ }^{7}$ Y. Tokunaga, S. Iguchi, T. Arima, and Y. Tokura, Phys. Rev. Lett. 101, 097205 (2008).

${ }^{8}$ A. Kirilyuk, A. V. Kimel, and T. Rasing, Rev. Mod. Phys. 82, 2731 (2010).

${ }^{9}$ S. Artyukhin, M. Mostovoy, N. P. Jensen, D. Le, K. Prokes, V. G. de Paula, H. N. Bordallo, A. Maljuk, S. Landsgesell, H. Ryll,
}

B. Klemke, S. Paeckel, K. Kiefer, K. Lefmann, L. T. Kuhn, and D. N. Argyriou, Nat. Mater. 11, 694 (2012).

${ }^{10}$ Y. Tokunaga, Y. Taguchi, T.-h. Arima, and Y. Tokura, Nat. Phys. 8, 838 (2012).

${ }^{11}$ D. Albrecht, S. Lisenkov, W. Ren, D. Rahmedov, I. A. Kornev, and L. Bellaiche, Phys. Rev. B 81, 140401 (2010).

${ }^{12}$ L. Bellaiche, G. Zhigang, and A. K. Igor, J. Phys.: Condens. Matter 24, 312201 (2012).

${ }^{13}$ R. M. Bozorth, V. Kramer, and J. P. Remeika, Phys. Rev. Lett. 1, 3 (1958).

${ }^{14}$ K. P. Belov, A. M. Kadomtseva, T. M. Ledneva, T. L. Ovchinnikova, and V. A. Timofeeva, JETP Lett. 2, 161 (1965).

${ }^{15}$ Y. K. Jeong, J.-H. Lee, S.-J. Ahn, and H. M. Jang, Solid State Commun. 152, 1112 (2012).

${ }^{16}$ V. N. Derkachenko, A. M. Kadomtseva, V. A. Timofeeva, and V. A. Khokhlov, JETP Lett. 20, 104 (1974).

${ }^{17}$ K. Yoshii, J. Solid State Chem. 159, 204 (2001).

${ }^{18}$ K. Yoshii, A. Nakamura, Y. Ishii, and Y. Morii, J. Solid State Chem. 162, 84 (2001).

${ }^{19}$ Y. Ren, T. T. M. Palstra, D. I. Khomskii, E. Pellegrin, A. A. Nugroho, A. A. Menovsky, and G. A. Sawatzky, Nature 396, 441 (1998). 
${ }^{20}$ A. V. Mahajan, D. C. Johnston, D. R. Torgeson, and F. Borsa, Phys. Rev. B 46, 10966 (1992).

${ }^{21}$ J. S. Jung, A. Iyama, H. Nakamura, M. Mizumaki, N. Kawamura, Y. Wakabayashi, and T. Kimura, Phys. Rev. B 82, 212403 (2010).

${ }^{22}$ J. G. Cheng, J. S. Zhou, J. B. Goodenough, Y. T. Su, Y. Sui, and Y. Ren, Phys. Rev. B 84, 104415 (2011).

${ }^{23}$ F. Hong, Z. Cheng, and X. Wang, Appl. Phys. Lett. 99, 192503 (2011).

${ }^{24}$ Y. Wang, S. Cao, M. Shao, S. Yuan, B. Kang, J. Zhang, A. Wu, and J. Xu, J. Cryst. Growth 318, 927 (2011).

${ }^{25}$ S. Yuan, Y. Wang, M. Shao, F. Chang, B. Kang, Y. Isikawa, and S. Cao, J. Appl. Phys. 109, 07E141 (2011).

${ }^{26}$ J. P. Perdew, J. A. Chevary, S. H. Vosko, K. A. Jackson, M. R. Pederson, D. J. Singh, and C. Fiolhais, Phys. Rev. B 46, 6671 (1992).

${ }^{27}$ G. Kresse and D. Joubert, Phys. Rev. B 59, 1758 (1999).

${ }^{28}$ G. Kresse and J. Hafner, Phys. Rev. B 47, 558 (1993).

${ }^{29}$ J. P. Perdew, K. Burke, and M. Ernzerhof, Phys. Rev. Lett. 77, 3865 (1996).
${ }^{30}$ J. P. Perdew, A. Ruzsinszky, G. I. Csonka, O. A. Vydrov, G. E. Scuseria, L. A. Constantin, X. Zhou, and K. Burke, Phys. Rev. Lett. 100, 136406 (2008).

${ }^{31}$ V. I. Anisimov, F. Aryasetiawan, and A. I. Lichtenstein, J. Phys.: Condens. Matter 9, 767 (1997).

${ }^{32}$ W. Sławiński, R. Przeniosło, I. Sosnowska, and E. Suard, J. Phys.: Condens. Matter 17, 4605 (2005).

${ }^{33}$ H. J. Monkhorst and J. D. Pack, Phys. Rev. B 13, 5188 (1976); P. E. Blöchl, O. Jepsen, and O. K. Andersen, ibid. 49, 16223 (1994).

${ }^{34}$ Y. B. Bazaliy, L. T. Tsymbal, G. N. Kakazei, A. I. Izotov, and P. E. Wigen, Phys. Rev. B 69, 104429 (2004).

${ }^{35}$ L. T. Tsymbal, V. I. Kamenev, Y. B. Bazaliy, D. A. Khara, and P. E. Wigen, Phys. Rev. B 72, 052413 (2005).

${ }^{36} \mathrm{~K}$. Momma and F. Izumi, J. Appl. Crystallogr. 41, 653 (2008).

${ }^{37}$ J. Bartolomé, E. Palacios, M. D. Kuz'min, F. Bartolomé, I. Sosnowska, R. Przeniosło, R. Sonntag, and M. M. Lukina, Phys. Rev. B 55, 11432 (1997). 